\title{
Factores socioeconómicos que influyen al estudiante en el emprendimiento universitario en la provincia de Tungurahua.
}

\author{
Socio-economic factors that influence the student in the university \\ entrepreneurship in the province of Tungurahua.
}

Lcdo. Padilla Martínez - Mario Patricio Mg. ${ }^{1}$, Efraín Velasteguí López Mg. ${ }^{2}$, Ana Lucia Quispe Otacoma. ${ }^{3}$, De La Cruz Martínez Lissette Vanessa. ${ }^{4}$, Moya Quishpe Katerine Elizabeth. ${ }^{5}$

Recibido: 01-09-2017 / Revisado 01-11-2017 Aceptado: 11-12-2017/ Publicado: 01-01-2018

\begin{abstract}
.
DOI: https://doi.org/10.33262/cienciadigital.v2i1.30

The research is based on the objective of identifying the socioeconomic factors that influence the student in university entrepreneurship in the province of Tungurahua, the universities that was taken as a basis for the development of research are: Technical University of Ambato (UTA), University (UTI), Pontifica Universidad Católica del Ecuador (PUCESA), institutions of higher education oriented to the formation of individuals with an entrepreneurial profile capable of creating their own companies and generating sources of employment and contribute to the development of the province and therefore of the country; theoretical, applying research methods based on a theoretical basis through the guiding thread, qualitative approach, field research, bibliographic, descriptive, correlational, showing that the socioeconomic factors involved in the projects are social, economic, political and cultural, influencing the student in the enterprise, determining that they are the main constraints that limit for a university student to undertake and achieve success.
\end{abstract}

\footnotetext{
${ }^{1}$ Universidad Tecnica de Amabto, Ciencias Administrativas, Ambato, Ecuador, mp.padilla@uta.edu.ec

${ }^{2}$ Revista cienfifica indexada Ciencia Digital, Ambato, Ecuador, luisefrainvelastegui@cienciadigital.org

${ }^{3}$ Universidad de las Fuerzas Armas- Latacunga; Departamento de Ciencias Económicas Administrativas y de Comercio, Cotopaxi, Ecuador ; alquishpe3@espe.edu.ec

${ }^{4}$ Universidad Tecnica de Amabto, Ciencias Administrativas, Ambato, Ecuador, lisv_1993@hotmail.com

${ }^{5}$ Universidad Tecnica de Amabto, Ciencias Administrativas, Ambato, Ecuador, katylyz@ hotmail.com
} 
Keywords: Factors, Socioeconomic, Social, Economic, Political, Cultural, Entrepreneurship, Types of Entrepreneurship.

\section{Resumen}

La investigación se basa en el objetivo de identificar los factores socioeconómicos que influyen al estudiante en el emprendimiento universitario en la provincia de Tungurahua, las universidades que se tomó como base para el desarrollo de la investigación son: Universidad Técnica de Ambato (UTA), Universidad Regional Autónoma de Los Andes (UNIANDES), Universidad Tecnológica Indoamérica (UTI), Pontifica Universidad Católica del Ecuador (PUCESA), instituciones de educación superior orientadas a la formación de individuos con perfil emprendedor capaces de crear su propia empresas y generar fuentes de empleo y contribuir con el desarrollo de la provincia y por ende del país; teórica aplicando métodos de investigación basados en una fundamentación teórica a través del hilo conductor, enfoque cualitativo, investigación de campo, bibliográfica, descriptiva, correlacional, evidenciando que los factores socioeconómicos que intervienen en los emprendimientos son social, económico, político, cultural, que influyen al estudiante en el emprendimiento, determinando que son los principales condicionantes que limitan para que un estudiante universitario pueda emprender y llegar al éxito .

Palabras Claves: Factores, Socioeconómicos, Sociales, Económicos, Políticos, Culturales, Emprendimiento, Tipos de Emprendimiento.

\section{Introducción.}

En la provincia de Tungurahua existen 4 universidades tanto públicas y privadas que apoyan a los emprendimientos universitarios en cada una de sus facultades dentro de las cuales se encuentran: Universidad Técnica de Ambato (UTA), Universidad Regional Autónoma de Los Andes (UNIANDES), Universidad Tecnológica Indoamérica (UTI), Pontifica Universidad Católica del Ecuador (PUCESA), enfocadas ayudar a los estudiantes universitarios a generar emprendimientos y a dinamizar la economía del país.

Para enfrentar la problemática en los estudiantes universitarios al emprender un negocio han surgido varias labores, tanto estatales, institucionales, corporativas, y sociales, surgiendo la figura de factores socioeconómicos orientados a beneficios medibles en la economía como en lo social (Fischel, 2013).

El objetivo que se planteó en el proyecto de investigación fue identificar los factores socioeconómicos que inciden en el éxito o fracaso del estudiante al iniciar un 
emprendimiento en la provincia de Tungurahua, fundamentar teóricamente y científicamente los factores socioeconómicos que influyen en el éxito o fracaso en los emprendimientos universitarios, Caracterizar la situación actual de los emprendimientos en las universidades a través de una investigación de campo, describir que factores socioeconómicos inciden al estudiante al iniciar un emprendimiento

Al momento de especificar los factores que inciden en el éxito o fracaso de un estudiante al realizar un emprendimiento, no es extraño hallar muchos problemas, debido a que dichos factores, socioeconómicos forman una red fuertemente entretejida que estacan a que un emprendimiento pueda nacer en el Ecuador (González, 2013). Siendo el emprendimiento universitario uno de los principales temas de interés de la entidad académica, de las direcciones de gobierno (Gurrero, Urbano, \& Ramos, 2016).

Los factores que obstaculizan a que los emprendimientos puedan surgir y llegar al éxito en el Ecuador se basan principalmente en factores tales como: El ámbito social, económico, político y cultural elementos críticos que impiden a la creación de nuevos emprendimientos o empresas, es así como dentro del ámbito regional el entorno no se ha percibido favorable por la inestabilidad política y demás amenazas en el ámbito financiero (Padilla, Quispe, Nogueira, \& Hernández, 2016). Donde los escenarios más perjudiciales para el apoyo de los emprendimientos en los estudiantes universitarios y personas en particular serian el apoyo financiero y políticas de gobiernos cambiantes. (Lasio,Virginia;Caicedo,Guido; Ordeñana, Xavier;Samaniego,Andrea, 2016)

En los factores sociales la enseñanza forma externalidades positivas para la sociedad. Lo que permite a que los adelantos y las nuevas preparaciones sean conocidos por otras sociedades favoreciendo a su progreso en movimientos comerciales. La educación ayuda a la disminución de las diferencias sociales y a la estabilidad social, creando así un ambiente más ventajoso para el emprendimiento. (Beatriz, 2015)

Existen factores que apoyan o afectan el rendimiento académico, es un tema que no sólo preocupa a las autoridades educativas, sino también a la sociedad en general. Donde se desarrollan destrezas, habilidades, conocimientos de los estudiantes universitarios, para formar futuros profesionales (Gonzalez, 2015).

Un aspecto principal del emprendimiento, es el dominio que posee sobre el progreso económico de un país a nivel mundial. Lo que implica desarrollo financiero, ya que nos referimos a implantar nuevos servicios, por lo que a su vez se creara más capital y se aumentara puestos de trabajo (Torroba, 2014).

Las políticas oportunista y discriminatorias, ejercidas por los países lujosos, imposibilitan que la mayoría de los países participe activamente en los cambios productivos y 
financieros; es decir, no alcanzan a globalizarse, y si lo hacen es simplemente a través de las transnacionales vecinas en sus economías (García N. , 2012).

El Régimen Emprende Ecuador fue creado por El Ministerio de Coordinación de la Producción, Empleo y Competitividad como estrategias de política nacional de emprendimiento. Su creación en la zona se dio mediante la vinculación de la segmento público y privado, revirtiéndole en un instrumento para viabilizar técnicas conducidas con al contexto emprendedor siendo una asociación de mercados (Barriel \& Otero, 2014).

Los factores culturales indican esencialidades de las conductas de los seres humanos como son algunos aspectos como la religiosidad, costumbres y tradiciones aportan un conjunto de significados que no se pueden esquivar en los estudios de las comunidades (Reyes, 2014).

\section{Métodos y materiales.}

Se utilizó un enfoque cualitativo lo que pretende captar el significado de las cosas, procesos, comportamientos (Padilla, Quispe, Nogueira, \& Hernández, 2016). El papel de la teoría es crucial, tanto en la perspectiva cuantitativa como cualitativa ya que una investigación sin un marco teórico o un marco de referencia pertinente sería una investigación aislada que puede conducir a la especulación o al establecimiento de juicios de valor, situación originada en múltiples falencias en la construcción o síntesis teórica como parte del proceso de investigación (Ruíz, 2012).

La investigación se maneja de acuerdo a la modalidad de campo con la intención de implicarse con la realidad, que existe en los emprendimientos universitarios fue través del contacto directo de los investigadores con los estudiantes universitarios para recolectar y registrar la información primaria, relativo al problema de estudio (Di Virgilio, y otros, 2014).

La investigación bibliográfica que permitió fundamentar el marco teórico y el tipo de investigación que se utilizo es la descriptiva utiliza para describir y detallar toda la información acerca de la problemática que existe en los factores socioeconómicos influyentes en el estudiante al realizar emprendimientos universitarios obteniendo resultados mediante las encuestas a realizarse, que servirán como guía para la investigación. (Silamani \& Guíra, 2015)

La población es el conjunto de elementos que serán vistos en la elaboración de un proyecto de indagación. Cada componente que forma la población se lo llama individuo (Vargas, 2013). Cálculo proporcional de la muestra para cada una de las universidades 
Tabla 1. Total estudiantes en porcentaje

\begin{tabular}{cccc}
\hline UNIVERSIDADES & \# DE ESTUDIANTES & PORCENTAJE & \# DE ENCUESTAS \\
\hline UTA & $14.222,00$ & $60,99 \%$ & 230 \\
UNIANDES & 3544 & $15,20 \%$ & 58 \\
UTI & $3.220,00$ & $13,81 \%$ & 52 \\
PUCESA & $2.331,00$ & $10,00 \%$ & 38 \\
TOTAL & $\mathbf{2 3 . 3 1 7 , 0 0}$ & $\mathbf{1 0 0}$ & $\mathbf{3 7 8}$ \\
\hline
\end{tabular}

Fuente: Universidades de Tungurahua

Elaborado por: Lissette de la Cruz, Katerine Moya

Una vez realizado los cálculos correspondientes se puede observar en la tabla 1 la distribución del número de encuestas que se deben aplicar a cada universidad de la provincia de Tungurahua. Realizando de tal manera el $100 \%$ de las encuestas que se encuentran distribuidas siendo la UTA la más alta con un porcentaje del 60.99\%, UNIANDES $15.20 \%$, UTI $13.81 \%$ y por último la PUCESA $10 \%$ cumpliendo en su totalidad el porcentaje de muestreo total.

\section{Resultados.}

La información producida mediante el análisis de las encuestas realizadas se examinó a través de las respuestas de los estudiantes universitarios.

\section{¿En qué Institución educativa usted estudia?}

Gráfico 1: Institución educativa

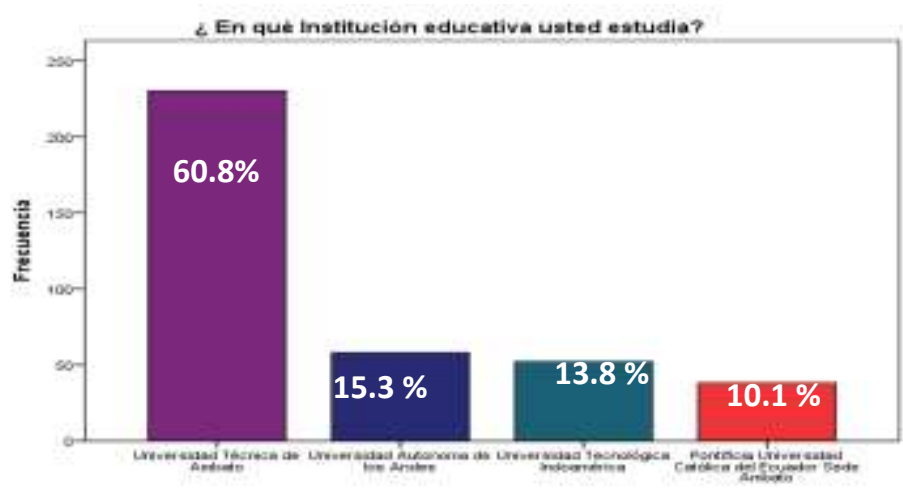

Fuente: Encuesta (2017)

De las 378 de las personas encuestadas, el 60.8\% son estudiantes de la Universidad Técnica de Ambato, el 15.3\% de la Universidad Autónoma de los Andes, el 13.8\% de la 
Universidad Tecnológica Indoamérica y el 10.1\% de la Pontificia Universidad Católica del Ecuador Sede Ambato, de acuerdo a la encuesta realizada se observa que la mayoría de estudiantes encuestados son de la Universidad Técnica de Ambato, sabiendo que el porcentaje de estudiantes es más alto y la Pontificia Universidad Católica del Ecuador Sede Ambato tiene una minoría de estudiantes.

\section{Señale el nivel de importancia del emprendimiento universitario en los siguientes ámbitos}

Grafico $\mathbf{N}^{\mathbf{0}}$ 2: Importancia del emprendimiento universitario

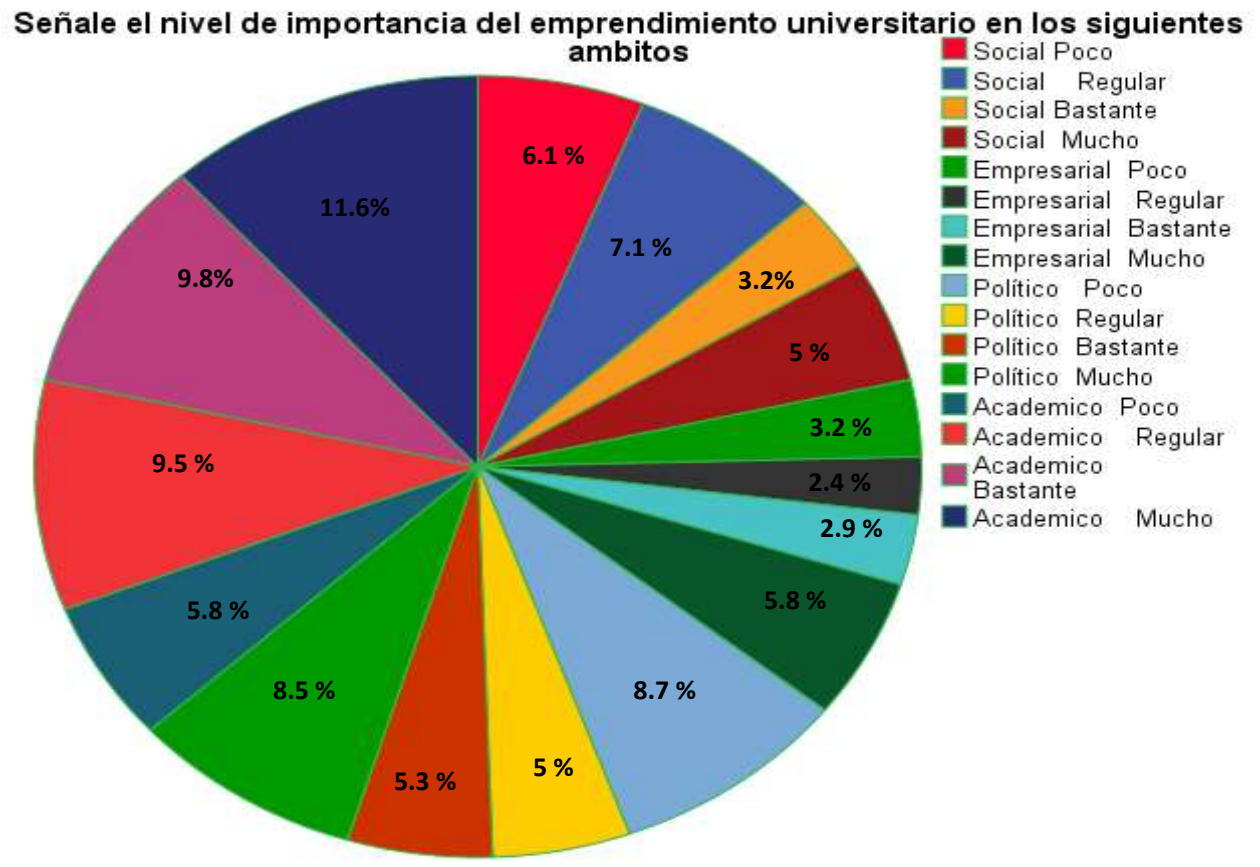

Fuente: Encuesta (2017)

Del total de las personas encuestada el $13.2 \%$ considera que el ámbito social tiene poco impacto sobre los emprendimientos, el $8 \%$ tiene mayor relevancia, el $5.6 \%$ considera que tiene poca importancia en el ámbito empresarial, el $8.7 \%$ alude que el ámbito influye de manera positiva a los emprendimientos universitarios, el $13.7 \%$ que el ámbito político no tiene mucha importancia, el $13.8 \%$ demuestra que si es importante al momento de realizar un emprendimiento, el $15.3 \%$ enseña que la educación influye poco al emprender un negocio, al contrario del $21.4 \%$ expone que el ámbito académico influye de manera positiva al momento de emprender un negocio. Los resultados analizados demuestran que los impactos de mayor importancia sobre los emprendimientos recaen en el ámbito académico 
como de mayor importancia mientras que el ámbito empresarial lo considera como poco importante en relación con los otros ámbitos puestos en estudio, fundamentando que el ámbito académico se encuentra realizando capacitaciones semestrales que ayudan a que los emprendimientos surjan de manera positiva.

Con respecto a la posibilidad de emprender, cuál cree que son los aspectos más interesantes a conocer. Señale la de mayor interés para usted.

Grafico No 3: Aspectos relevantes de los factores socioeconómicos

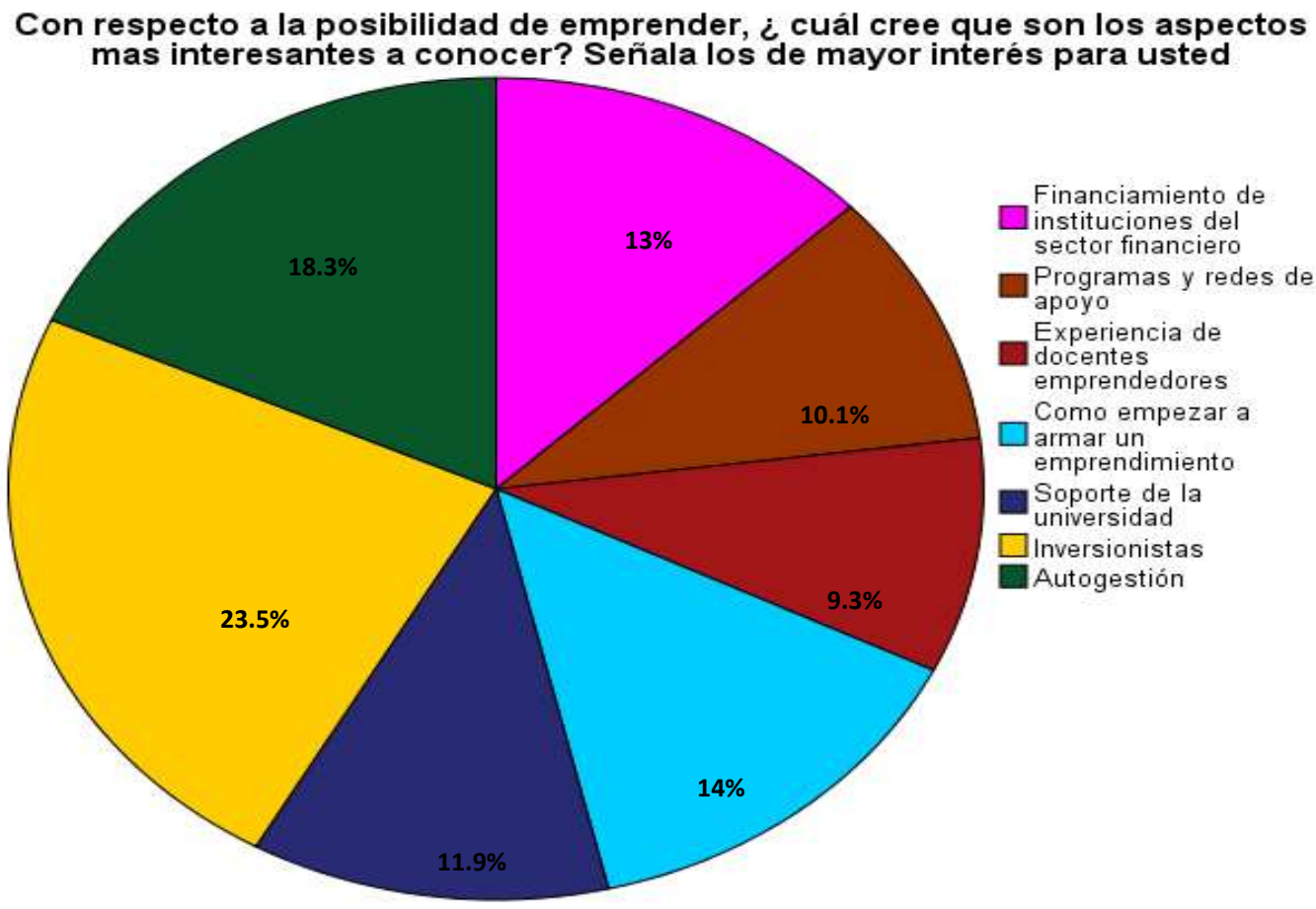

Fuente: Encuesta (2017)

Del total de los estudiantes encuestados, el $23.5 \%$ que son los Inversionistas, el $18.3 \%$ que es la autogestión, el 14\% alude que el cómo empezar a armar un emprendimiento es muy importante de conocer, $13 \%$ señala que el financiamiento es uno de los aspectos más interesantes a conocer al iniciar un emprendimiento, el $11.9 \%$ indica que es el soporte de la universidad, el $10.1 \%$ que son los programas y redes de apoyo, el $9.3 \%$ la experiencia de docentes emprendedores. Considerando como base fundamental cada una de las repuestas se puede deducir que los aspectos con relevancia son los inversionistas es decir deben conocer cuáles de las instituciones públicas o privadas se interesan por idea innovadora y la autogestión porque la mayoría de los estudiantes pueden financiarse sus emprendimientos. 


\section{Califique los factores socioeconómicos que influyen al estudiante en el emprendimiento universitario.}

Grafico No 4: Factores socioeconómicos

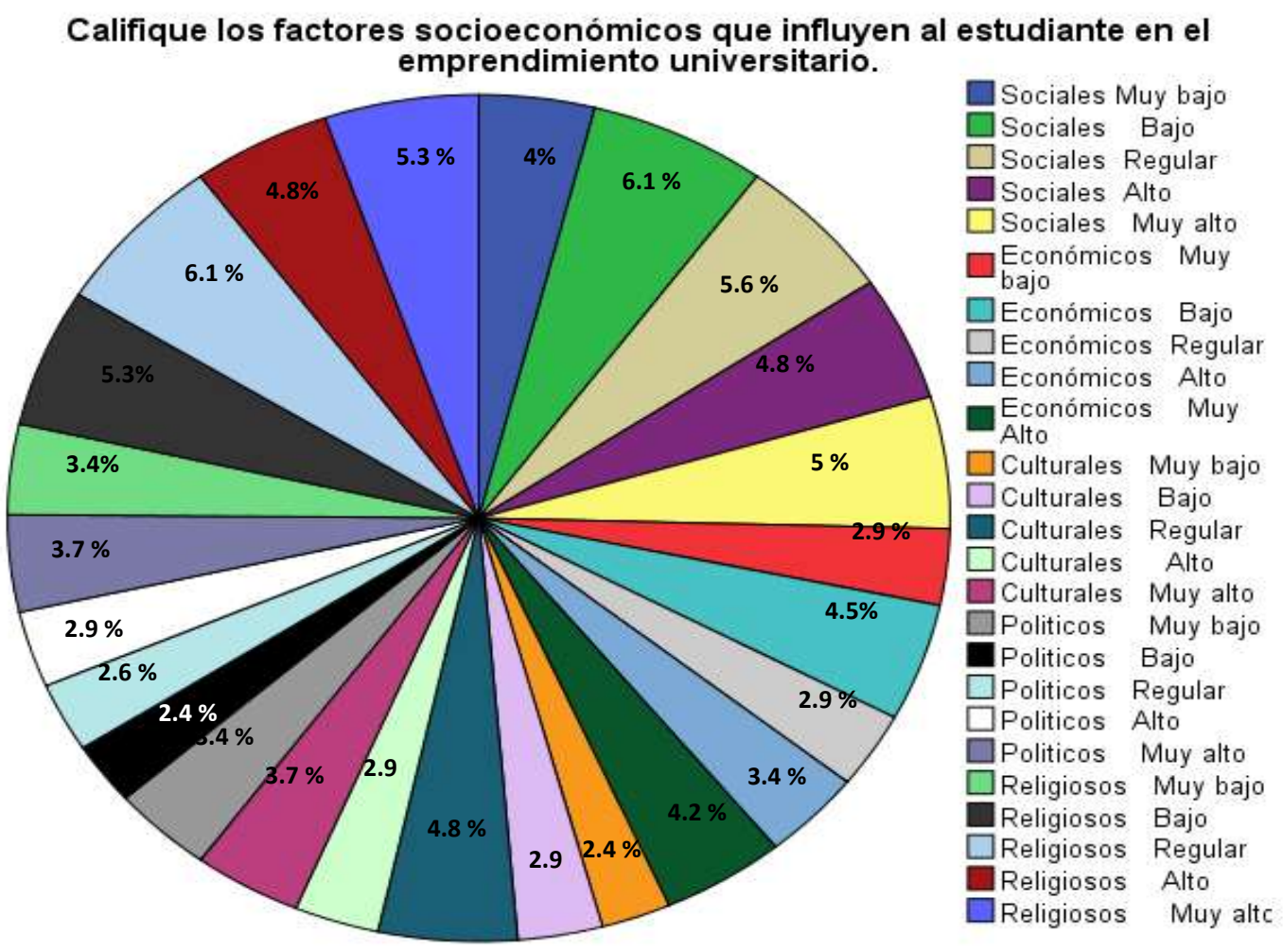

Fuente: Encuesta (2017)

Del total de las personas encuestas el $15.7 \%$ expone que los factores sociales influyen poco en los estudiantes, el 9.8\% menciona que existe un índice alto de que lo social influya al realizar un emprendimiento, el $14.8 \%$ indica que el factor religioso no tiene mucha importancia al momento de emprender, y el 9.7\% considera que tiene una relevancia muy alta el $10.3 \%$ indica que la economía tiene un bajo índice de afectación a los emprendimientos, el $7.6 \%$ señala que la economía es la causante para que los estudiantes no puedan emprender, el $10.1 \%$ considera que el factor cultural recae en un bajo porcentaje para que el estudiante emprendan mientras que el 6.6\% coinciden que tienen una alta influencia, el $8.4 \%$ alude que el factor político es el que interviene poco en el emprendimiento y el $6.6 \%$ considera que la política tiene un alto índice de intervención en los emprendimientos. De tal manera que los resultados expuestos arrojan que el factor socioeconómico que mayor influencia tiene sobre los emprendimientos es el social seguido religioso, económico y político. 


\section{Califique las principales limitantes que tiene un estudiante para iniciar un emprendimiento universitario.}

Grafico No 5 Limitantes de los estudiantes

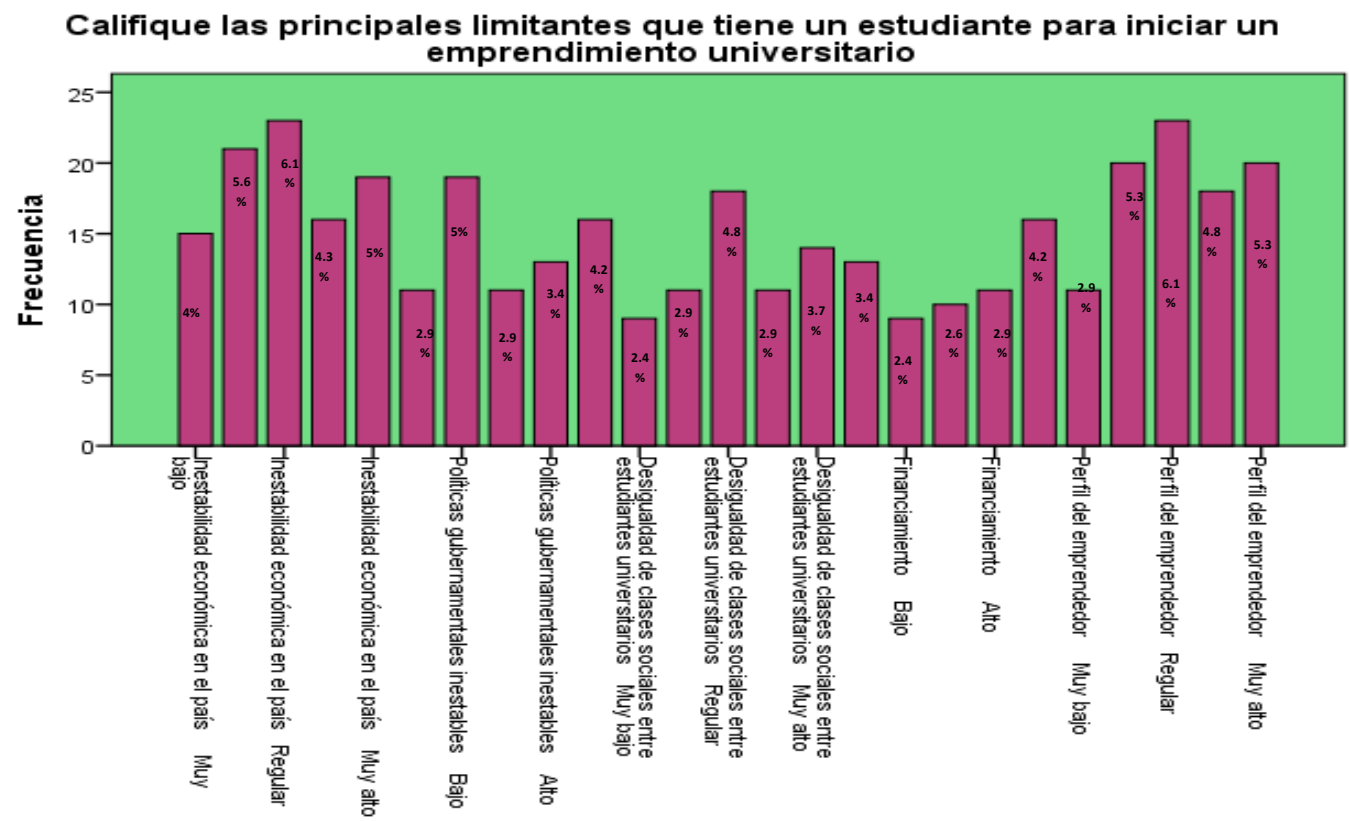

Fuente: Encuesta Estudiantes Universitarios de la Provincia de Tungurahua

De las personas encuestadas el $15.7 \%$ indica que la inestabilidad económica limita poco al estudiante para iniciar un emprendimiento, el 9.2\% señala que tiene un porcentaje alto $\mathrm{d}$ afectación, y el $10.8 \%$ considera que son las políticas gubernamentales inestables que afectan influyen poco en el emprendimiento y el $7.6 \%$ de los encuestados señalan que políticas gubernamentales inestables tienen un alto índice de influencia, el $10.1 \%$ señala que es muy baja la influencia de las desigualdad de las clases sociales entre estudiantes universitarios que influyan en el emprendimiento, el $6.6 \%$ considera que la desigualdad de las clases sociales tienen una influencia alta, el $14.3 \%$ alude que el perfil emprendedor influye poco y el $10.1 \%$ tienen un alto índice de influencia y el $8.4 \%$ señala que es el financiamiento y el $7.1 \%$ menciona que el financiamiento influye en un alto porcentaje al crear un emprendimiento. Con respecto a los datos analizados se considera que los limitantes que tiene los estudiantes de la provincia de Tungurahua para iniciar con su emprendimiento se enfocan primordialmente en que los estudiantes no tienen un alto perfil emprendedor seguido de la inestabilidad económica en el país que existe en la actualidad ya que son los causantes para que el estudiante pueda iniciar con su emprendimiento ya que temen a tener fracaso y no llegar al éxito cual ha sido su objetivo principal. 


\section{DISCUSIÓN}

A través de la investigación se pudo identificar que los factores socioeconómicos de gran impacto positivo y negativo en los emprendimientos universitarios son los siguientes, los datos estadísticos de fiabilidad demuestran, que en la tabla 1 la columna 1 indica el número de pregunta, la columna 2 indica el total de las personas encuestadas que son 378 , la columna 3 es la representación de la media la cual señala que tenemos una media total de 63.27, la desviación típica o estándar mide el grado de dispersión que es de 22.82 y su varianza 521.065, la cual permitió identificar las preguntas que responden a la problemática planteada en el proyecto.

Tabla 2. Estadísticos de Fiabilidad

\begin{tabular}{|c|c|c|c|c|}
\hline Preguntas & $\mathbf{N}$ & Media & Desv. típ. & Varianza \\
\hline ¿En qué Institución educativa usted estudia? & 378 & 1,73 & 1,038 & 1,078 \\
\hline ¿A qué etnia pertenece? & 378 & 2,03 & 1,161 & 1,349 \\
\hline $\begin{array}{l}\text { ¿Cree que pertenecer a una etnia afectaría al iniciar un } \\
\text { emprendimiento? }\end{array}$ & 378 & 2,88 & 1,296 & 1,679 \\
\hline ¿En su universidad realizan capacitaciones de emprendimiento? & 378 & 1,58 & 0,494 & 0,244 \\
\hline ¿Como lo hace? & 378 & 3,94 & 1,349 & 1,819 \\
\hline $\begin{array}{l}\text { ¿Considera usted que el emprendimiento universitario es } \\
\text { importante? }\end{array}$ & 378 & 1,18 & 0,385 & 0,148 \\
\hline $\begin{array}{l}\text { Con respecto a la posibilidad de emprender, ¿cuál cree que son } \\
\text { los aspectos más interesantes a conocer? Señala los de mayor } \\
\text { interés para usted }\end{array}$ & 378 & 4,46 & 2,042 & 4,169 \\
\hline $\begin{array}{l}\text { ¿Considera usted que las instituciones financieras apoyan a los } \\
\text { emprendimientos en la provincia de Tungurahua? }\end{array}$ & 378 & 1,41 & 0,492 & 0,242 \\
\hline ¿De qué tipo? & 378 & 1,74 & 0,442 & 0,195 \\
\hline $\begin{array}{l}\text { ¿Considera usted que los factores socioeconómicos limitan al } \\
\text { estudiante universitario para iniciar un emprendimiento? }\end{array}$ & 378 & 2,27 & 1,152 & 1,328 \\
\hline $\begin{array}{l}\text { Señale el nivel de importancia del emprendimiento universitario } \\
\text { en los siguientes ámbitos }\end{array}$ & 378 & 9,78 & 4,869 & 23,712 \\
\hline $\begin{array}{l}\text { Califique los factores socioeconómicos que influyen al } \\
\text { estudiante en el emprendimiento universitario. }\end{array}$ & 378 & 12,89 & 7,812 & 61,029 \\
\hline $\begin{array}{l}\text { Califica cómo influye el estrato socioeconómico en los } \\
\text { emprendimientos universitarios }\end{array}$ & 378 & 4,48 & 2,678 & 7,173 \\
\hline $\begin{array}{l}\text { Califique las principales limitantes que tiene un estudiante para } \\
\text { iniciar un emprendimiento universitario }\end{array}$ & 378 & 12,91 & 7,785 & 60,602 \\
\hline Suma & 378 & 63,2751 & 22,82684 & 521,065 \\
\hline $\mathrm{N}$ válido (según lista) & 378 & & & \\
\hline
\end{tabular}

Fuente: Programa Spss

Elaborado por: Lissette de la Cruz, Katerine Moya 
En la tabla 1 se identifica las respuestas a las encuestas ya realizadas y se destacan las siguientes:

\section{Califique las principales limitantes que tiene un estudiante para iniciar un emprendimiento universitario}

En la tabla 2 se puede observar que los limitantes para realizar un emprendimiento son la inestabilidad económica con $15.1 \%$, Perfil del emprendedor con un $12.5 \%$, políticas gubernamentales $10.4 \%$, Desigualdad de clases sociales $9.7 \%$ y el financiamiento es $8.2 \%$. Tomando en cuenta que la inestabilidad económica en el país tiene un porcentaje bajo de limitación a los estudiantes, las políticas gubernamentales no afectan ya que si existen leyes que apoyan al emprendimiento como es el Innova Ecuador.

Tabla 3. Limitantes del emprendimiento

\begin{tabular}{lc}
\hline \multicolumn{1}{c}{ Limitantes del emprendimiento } & Porcentaje \\
\hline Inestabilidad económica en el país $\quad$ Muy bajo & 15.1 \\
Políticas gubernamentales inestables $\quad$ Muy alto & 10.4 \\
Desigualdad de clases sociales entre estudiantes universitarios & 9.7 \\
Muy bajo & 8.2 \\
Financiamiento Muy abajo & 12.5 \\
Perfil del emprendedor Muy bajo & \\
\hline
\end{tabular}

Fuente: Programa Spss

Elaborado por: Lissette de la Cruz, Katerine Moya

\section{Califique los factores socioeconómicos que influyen al estudiante en el emprendimiento universitario.}

En la tabla 3 señala que los factores socioeconómicos que influyen en el emprendimiento son los factores religiosos con un $5.1 \%$, sociales que tienen un $4.8 \%$, económicos con un $4.1 \%$, los culturales y políticos tienen un porcentaje de 3.6\%. Los factores sociales influyen de manera positiva, el factor religioso afecta ya que los estudiantes universitarios tienen diferentes ideologías y eso causa que haya problemas al momento de emprender. 
Tabla 4. Factores socioeconómicos

\begin{tabular}{lc}
\hline \multicolumn{1}{c}{ Indicador } & Porcentaje \\
\hline Sociales, Muy alto & 4,8 \\
Económicos, Muy Alto & 4,1 \\
Culturales, Muy alto & 3,6 \\
Políticos, Muy alto & 3,6 \\
Religiosos, Muy alto & 5,1 \\
\hline
\end{tabular}

Fuente: Programa Spss

Elaborado por: Lissette de la Cruz, Katerine Moya

\section{Señale el nivel de importancia del emprendimiento universitario en los siguientes ámbitos}

En la tabla 4 se indica el nivel de importancia del emprendimiento universitario en los siguientes ámbitos, el nivel académico es de mayor relevancia ya que su porcentaje es de $11.2 \%$ seguido del ámbito político que es el $8.2 \%$, el ámbito empresarial con el $5.6 \%$ y lo social con un $4.8 \%$. El ámbito que más influye es el académico ya que el nivel d estudio que se tiene ayuda a emprender un negocio y en el ámbito social las clases sociales no intervienen en el emprendimiento universitario.

Tabla 5. Importancia de los factores

\begin{tabular}{lr}
\hline \multicolumn{1}{c}{ Indicador } & Porcentaje \\
\hline Social, Mucho & 4,8 \\
Empresarial, Mucho & 5,6 \\
Político, Mucho & 8,2 \\
Académico, Mucho & 11,2 \\
\hline
\end{tabular}

Fuente: Programa Spss

Elaborado por: Lissette de la Cruz, Katerine Moya

En la tabla 5 se indica que los factores que influyen de manera positiva y negativa que surgieron a través de la presente investigación y sin indicar que sean los únicos, pero si los 
más frecuentes que están considerados por los estudiantes universitarios de la Provincia de Tungurahua y por el marco teórico realizado, son los siguientes:

Tabla 6. Diagnostico de los factores socioeconómicos

\section{SOCIALES}

La enseñanza forma características positivas para los De acuerdo al análisis realizado el $4.1 \%$ de las personas estudiantes, tomando en cuenta que nivel de indica que si ayuda la economía en los educación ayuda a que se eliminen las diferencias sociales observando que al ámbito académico tiene un $11.2 \%$.

Siendo muy alto su porcentaje lo que ayudara de una manera positiva al emprender un negocio.

El estrato social de nivel medio afecta de manera positiva en los estudiantes, ya que ellos poseen mayor conocimiento que podrían ayudar a las personas que carecen de conocimientos científicos.

\section{POLÍTICOS}

Desde la perspectiva de los estudiantes encuestados la política y las leyes planteadas en el Ecuador la política influye de una manera positiva con el 3.6\% a que se pueda realizar emprendimientos, más bien los favorece con el fin de generar empleo. Existen instituciones tanto públicas como privadas que ayudan a fomentar el emprendimiento. Innova Ecuador es una de las políticas planteadas por el gobierno ya que otorga líneas de créditos juntamente con la CFN, BanEcuador, MIPRO, Cooperativa de Ahorro y Crédito 29 de octubre, Cooperativa de Ahorro y crédito Cámara de Comercio de Ambato, Siembra Futuro, entre otras, las mismas que apoyan a mejorar los escenarios y contenidos de las micro, pequeñas y medianas empresas de forma asociada o individual emprendimientos universitarios, Financiamiento: Es de suma importancia ya que se puede definir y escalar los emprendimientos universitarios, tomando en cuenta que no es un limitante en el emprendimiento universitario También, sirve para ampliar los planes y convertirlos en realidad en este caso los estudiantes universitarios se ven en la necesidad de fundar nuevos lazos que les contribuyen con información, que permitirán el camino a potenciales consumidores y mercados, en este aspecto el financiamiento afecta a los estudiantes universitarios de una forma positiva ya que la mayoría contesto que si existen instituciones financieras tanto públicas como privadas que apoyan a las personas con montos de dinero altos para poder emprender sus negocios. Autogestión: este aspecto al igual que el financiamiento tiene una relevancia alta ya que algunos estudiantes respondieron que ellos tienen sus recursos propios para poder crear su empresa ya que algunos de ellos viene de una clase socioeconómica alta, la misma que les permite crear sus negocios sin ayuda de ninguna institución financiera.

\section{CULTURALES}

En los factores culturales el porcentaje es de 3.6\% teniendo un mínimo de afectación en el emprendimiento se puede decir que en este factor influye la religión con un $5.1 \%$ por lo que a través del análisis se concluye que la etnia no siempre influye negativamente al momento de realizar un emprendimiento, de acuerdo a la recolección de datos se obtuvo que las personas de etnias mestiza $50.3 \%$, indígena $11.4 \%$, blanca $23 \%$ y afro ecuatoriana $15.3 \%$ pueden crear empresas sin que eso afecta a ninguna persona. Otro factor que se recalca es la religión ya que se transmite a través de la cultura, en la presente investigación los estudiantes mencionan que la religión $5.1 \%$ influye de manera positiva y negativa ya que todos no comparten la misma ideología. Lo que puede causar problemas al momento de realizar emprendimientos universitarios.

\footnotetext{
Fuente: investigación propia

Elaborado por: Lissette de la Cruz, Katerine Moya
} 


\section{Conclusiones.}

- Una vez realizado el proceso investigativo en el proyecto titulado "Modelos de Gestión de Emprendimiento Universitario" mediante la resolución: 0910-CUP2016, con el tema Factores socioeconómicos que influyen al estudiante en el emprendimiento universitario en la provincia de Tungurahua, se utilizó la investigación bibliográfica con el criterio de diferentes autores lo que permitió elaborar la fundamentación teórica del proyecto culminado, cumpliendo de tal forma con el primer objetivo planteado que es fundamentar teóricamente y científicamente los factores socioeconómicos que influyen en el éxito o fracaso de los emprendimientos universitarios para ello se realizó el hilo conductor como un modelo teórico a seguir.

- Para analizar la situación actual de los factores socioeconómicos de los emprendimientos universitarios en la provincia de Tungurahua se basó en un enfoque cualitativo, describiendo las cualidades de las dos variables que se analizaron, también se basó en la modalidad de investigación de campo en la cual se aplicó la encuesta y se procedió al análisis e interpretación de resultados.

- Del diagnóstico de los factores socioeconómicos se determinó que los componentes que influyen de forma positiva y negativa en los emprendimientos, son los siguientes: factor social $4.8 \%$ interviene el nivel de estudio de las diferentes clases sociales de nivel alto ya que ayudan a emprender su negocio, factor económico $4.1 \%$ en este el financiamiento de instituciones tanto públicas como privadas ayuda a la creación de negocios, $3.6 \%$ en las políticas y leyes establecidas por el gobierno ayudan a crear sitios de trabajo los cuales generaran empleos , 3.6\% en lo cultural, $5.1 \%$ en la religión ya que afecta porque los estudiantes tienen diferentes ideologías.

\section{Referencias bibliográficas.}

- Barriel, G., \& Otero, A. (2014). Diagnóstico para valorar la introducción de un nuevo servicio. . Diagnóstico para valorar la introducción de un nuevo servicio. , 85-93.

- Beatriz, J. (2015). Factores macroeconómicos que estimulan el emprendimiento. 51.

- Di Virgilio, M., Cecilia, F., Carolina, N., Alejandra, N., Carolina, P., \& Gabriela, P. (2014). Competencias para el trabajo de campo cualitativo: formando investigadores en Ciencias Sociales. Revista Argentina de Sociología, 90-110. 
- Fischel, A. (2013). Factores empresariales. Red Latinoamericana de Universidades por el emprendedurismo social .

- García, N. (2012). Globalización: aspectos políticos, económicos. Revista de Ciencias Sociales, 721-726.

- Gonzalez, E. (2015). Factores de contexto socieconomico y eduactivos en estudiantes de nivel superior. Interamerican Journal of Psychology, 412.

- González, J. (2013). Estudio sobre factores contexto en estudiantes universitarios para conocer por qué unos tienen éxito mientras otros fracasan. Revista Intercontinental de Psicología y Educación, 135-154.

- Gurrero, M., Urbano, D., \& Ramos, A. (2016). Perfil Emprendedor del Estudiante Universitario. RedEmprendia.

- Lasio,Virginia;Caicedo,Guido; Ordeñana, Xavier;Samaniego,Andrea. (2016). Graduate School of Management. ESPAE (Global Entrepreneurship Monitor GEM).

- Padilla, M., Quispe, A., Nogueira, D., \& Hernández, A. (2016). Diagnóstico y perspectivas de fomento del emprendimiento como instrumento de desarrollo. Ingeniería Industrial, 91-103.

- Reyes, M. (2014). Factores culturales y desarrollo cultural comunitario. Madrid.

- Ruíz, J. (2012). Metodología de la investigación cualitativa. Bilbao.

- Silamani, J., \& Guíra, A. (2015). Utilidad y tipos de revisión de literatura. SciElo, 292.

- Torroba, L. (2014). Factores claves en el emprendimiento. Madrid.

- Vargas, A. (2013). Estadística descriptiva e inferencial. Murcia.

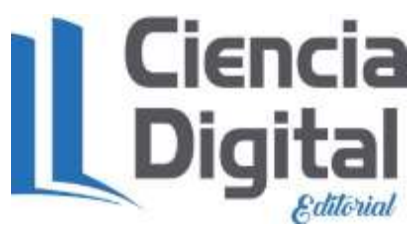




\section{Para citar el artículo indexado.}

Padilla M., Velasteguí E., Quispe A., De La Cruz L. \& Moya K. (2018). Factores socioeconómicos que influyen al estudiante en el emprendimiento universitario en la provincia de Tungurahua. Revista electrónica Ciencia Digital 2(1), 441-450. Recuperado desde:

http://www.cienciadigital.org/revistascienciadigital2/index.php/CienciaDigital/article/view/ $30 / 30$

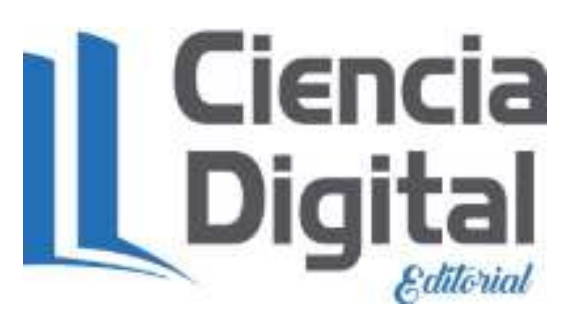

El artículo que se publica es de exclusiva responsabilidad de los autores y no necesariamente reflejan el pensamiento de la Revista Ciencia Digital.

El articulo queda en propiedad de la revista y, por tanto, su publicación parcial y/o total en otro medio tiene que ser autorizado por el director de la Revista Ciencia Digital.
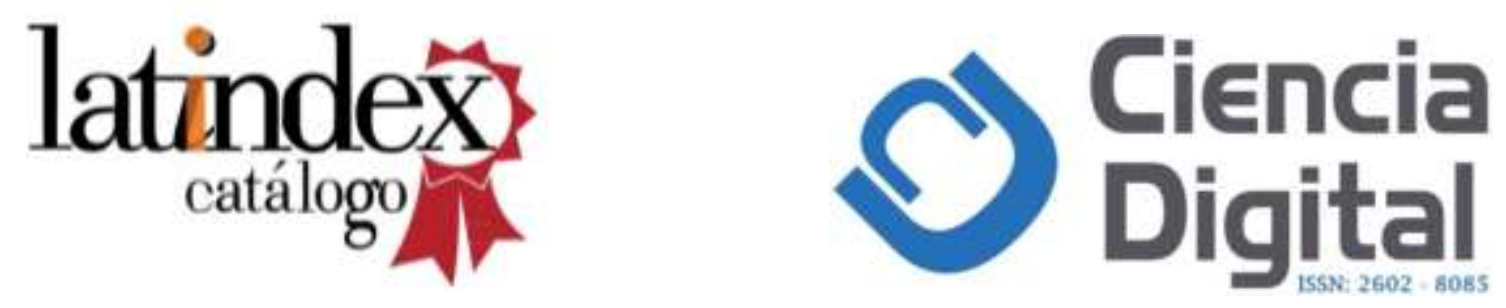